\title{
Investigating the Dynamic Tensile Response of Lean Duplex Stainless Steel and the Effects of Radial Waves Using the Recovered Plat-Impact Experiment
}

\author{
Ali A.H. Ameri ${ }^{*}$, Andrew D. Brown ${ }^{l}$,Zakaria Quadir ${ }^{2}$, Paul J. Hazell ${ }^{l}$, Con Logos ${ }^{3}$ and Juan P. Escobedo-Diaz ${ }^{l}$ \\ ${ }^{1}$ School of Engineering and Information Technology, UNSW, Canberra, ACT 2600, Australia \\ ${ }^{2}$ Microscopy and Microanalysis Facility (MMF), John de Laeter Centre (JdLC), Curtin University, Perth, WA 6102, Australia \\ ${ }^{3}$ Outokumpu, Laverton North, VIC 3026 Australia
}

\begin{abstract}
Lean Duplex Stainless Steel 2404 (LDX2404) is the newest commercial type of duplex stainless steels, recently introduced by Outokumpu ${ }^{\circledR}$. This paper investigates the dynamic tensile failure (spall strength) of this new alloy under different peak stresses (4.5 GPa to $8.2 \mathrm{GPa}$ ) via the recovered plate-impact experiment using a single stage gas gun. The microstructural investigation for the as-received and soft recovered post-shock material conditions were accomplished using Electron Backscatter Diffraction (EBSD) and optical microscopy. The effect of the radial release waves and the effectiveness of using momentum trap rings on the spall strength and the spall damage during the impact experiment were studied. Results indicate that LDX2404 experienced spall damage for the entire range of tested peak stresses. The spall strength was not affected by the implementation of momentum trapping rings whereas; the damage content was highly influenced. The spall damage of LDX 2404 exists parallel to the phase boundary interfaces, which are normal to the impact direction. Grain orientation, based on Taylor factor values, was not controlling the voids nucleation and voids growth.
\end{abstract}

\section{Introduction}

Duplex stainless steels (DSSs) are dual phase alloys with equal volume fractions of ferrite and austenite phases. These alloys have attractive mechanical properties (yield stress, ultimate tensile stress, ductility and corrosion resistance) [1]. Conventional types of DSSs (e.g. LDX2205) have limited usage because of unstable and expensive costs. This is because of the high content of expensive alloying elements ( $\mathrm{Ni}$ and $\mathrm{Mo}$ ) [2]. Recently, Lean Duplex Stainless Steel (LDX) was introduced as a new type of DSSs, aiming to cut volatile costs by partially replacing nickel with nitrogen and molybdenum with manganese and chromium [1,2].

LDX2404 is the most recent alloy which has been introduced to the lean duplex family by Outokumpu ${ }^{\circledR}$ [3]. This alloy has the highest yield stress and ultimate tensile stress of the commercially available DSSs. .LDX2404 was designed to be used for a wide range engineering applications where high strength and high corrosion resistance are required such as storage tanks, piping system and structural components. For these sorts of applications extreme loading conditions (high strain rates and temperature) are highly possible. Consequently, understanding the mechanical response of this new alloy at these conditions is important. However, the dynamic response of LDX2404 and how its phases interact at high strain rates have not been studied yet.
Spall strength (dynamic tensile strength) is one of the mechanical properties of the materials; it is estimated via plate impact experiment from measuring the free surface velocity. In this experiment, materials are subjected to a state of tensile stresses due to the interactions of the rarefaction fans $[4,5]$. If the tensile stresses exceed the material strength, the material develops spall failure as nucleation, growth, coalescence of voids depending on the test conditions [4]. During the plate impact experiments, other waves (radial release and edge waves) generate, and they can affect the failure mode and material microstructure ${ }^{[6]}$. The spall failure of DSSs is complex due to the complexity of their microstructure, ferrite and austenite phases have different crystallographic structures and these structures usually develop different types of failure during plate impact experiments as reported by Escobedo-Diaz et al. [7]. In this paper, the spall strength and failure of LDX2404 is studied using a soft-recovery plate-impact experiment. Furthermore, the effects of radial release waves, which are generated at the circumference of the flyer, on the spall strength and damage content were also explored.

\section{Materials and experimental methods}

A hot rolled coil-plate of LDX2404 with a $6 \mathrm{~mm}$ thickness produced by Outokumpu ${ }^{\circledR}$ was used in this 
study. The plate was annealed at $1040{ }^{\circ} \mathrm{C}$ followed by rapid cooling using forced air and water (i.e. quenching). The bulk chemical composition of the used material is shown in Table 1.

Table 1. Chemical composition of the used LDX2404.

\begin{tabular}{|c|c|c|c|c|c|c|c|}
\hline Element & $\mathrm{C}$ & $\mathrm{Si}$ & $\mathrm{Mn}$ & $\mathrm{P}$ & $\mathrm{Cr}$ & $\mathrm{Ni}$ & $\mathrm{Mo}$ \\
\hline $\mathrm{wt} \%$ & 0.02 & 0.42 & 2.93 & 0.03 & 24.12 & 3.66 & 1.61 \\
\hline Element & $\mathrm{Cu}$ & $\mathrm{N}$ & $\mathrm{Fe}$ & \multicolumn{5}{|l}{} \\
\hline $\mathrm{wt} \%$ & 0.34 & 0.27 & $\mathrm{Bal}$ & \multicolumn{3}{|l}{} \\
\hline
\end{tabular}

Electron Backscatter Diffraction (EBSD) scans were performed using a Field Emission Scanning Electron Microscope (FESEM) (TESCAN MIRA3). Optical microscopy (OM) images were taken using Zeiss microscope AXIO. Samples were prepared for microstructural analyses by following standard metallographic procedures up to vibratory polishing with $0.02 \mu \mathrm{m}$ non-crystallizing colloidal silica suspension.

Plate-impact experiments were performed using a 70 $\mathrm{mm}$ diameter bore, $13 \mathrm{~m}$ length single-stage light gas gun. The impact occurs by accelerating a flyer with 60 $\mathrm{mm}$ diameter, attached to a sabot made of Polyurethaneresin foam, towards the targets holder as shown in Fig. (1). Both the flyer and targets holder were made of LDX2101, with the flyer measuring half the thickness $(1.4 \mathrm{~mm})$ of the target assembly $(2.8 \mathrm{~mm})$ to develop a centralized spall plane within the samples. The targets holder contains samples (targets) and momentum trap rings, which are also made of LDX2404. Samples and the inner ring (Fig. (1)) were tapered with a $7^{\circ}$ angle to facilitate release from the target assembly during the impact. Samples, rings and the targets holder were assembled using a universal testing machine by applying a compressive force of around $2.5 \mathrm{kN}$. Tolerances for all the assembled parts were interference fits/locational interference. Prior to assembly, all parts were lubricated with molybdenum disulphide $\left(\mathrm{MoS}_{2}\right)$ to facilitate in-situ separation of the samples and to remove any air voids. After assembling all parts, the target assembly were machined down to the final thickness with a tolerance for the samples' faces parallelism of about $5 \mu \mathrm{m}(0.4$ $\mathrm{mrad})$. The assembly was aligned in the gas gun using a laser central to the bore, resulting in less than $3 \mathrm{mrad}$ error in the impacts. Free surface velocity was measured using Photon Doppler Velocimetry (PDV) as shown in Fig. (1).

The aim of the flyer-target plate impact experiment is imparting a plastic shock wave with uniaxial strain conditions on the targets. However, during the experiment several types of waves may exist depending on the testing geometry [8-9]. Understanding complex wave interactions is critical to designing experiments in an appropriate way. Fig. (2) shows the possible waves that generate after the impact happens. Once the impact takes place, the sample is subjected to a uniaxial strain wave. Upon reaching the rear free surface, this wave reflects as a rarefaction fan and interacts with a similar rarefaction fan reflected from the flyer plate, resulting in large tensile stresses for the duration of wave interaction. Additionally, radial release waves at the circumference of the flyer propagate after impact, which reach to the edges of the target holder and reflected back as edge waves.

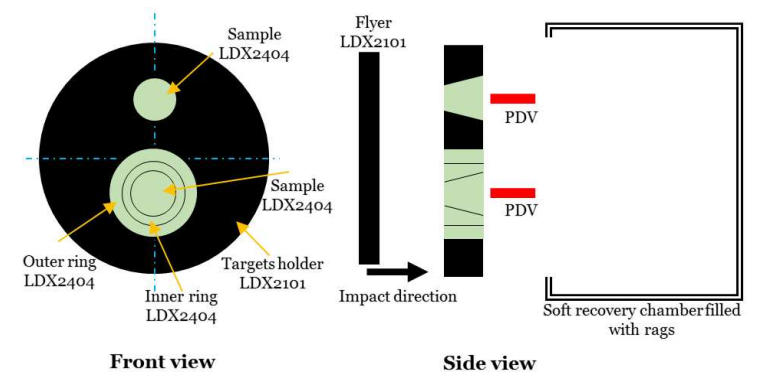

Fig. 1. Experimental configuration used for soft recovery of plat impact experiments.

The edge waves are typically not as critical for spall damage studies as the radial release waves may arrive in the sample well after spall has occurred. Many studies have elucidated the effect of radial release waves during shock experiments where these radial waves can compress the sample and cause significant changes in the microstructure $[6,10]$. These studies suggested using momentum trap rings to prevent/mitigate the effects of radial release waves. In this study, we explored the effectiveness of using momentum trap rings in the plateimpact experiment. We designed the rings based on the approach suggested in Orava and Wittman [11].

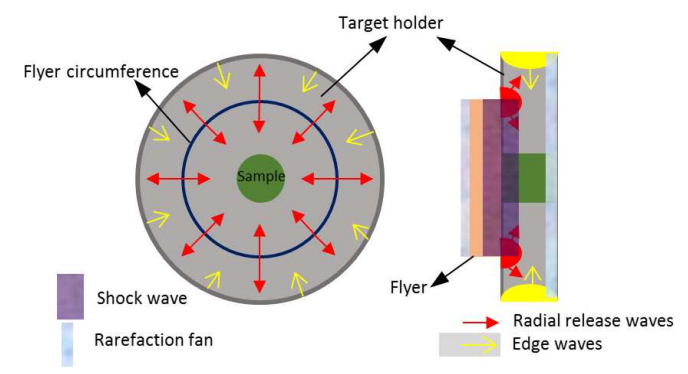

Fig. 2. Possible types of stress waves that generate during plate impact test.

The width of the ring (w) was estimated using Eq.(1), where the width of the ring should be large enough to trap the radial release waves until the rarefaction fans interact inside the targets (i.e. until spall damage occurs).

$$
w \geq\left(\frac{T_{f}}{U_{s}}+\frac{T_{f}}{C_{o f}}+\frac{T_{t}}{C_{o t}}\right) \times C_{l t}
$$

where $T_{f}, T_{t}, U_{s}, C_{o f}, C_{o t}$ and $C_{l t}$ are the thickness of the flyer, thickness of the targets holder, shock wave speed, bulk sound speed in the flyer material, bulk sound speed in the target material and longitudinal sound speed in the ring material, respectively. The above equation is based on the assumption that the rings are made from the same material of the sample (target). Based on Eq. (1), the largest width for the ring was estimated to be $7.79 \mathrm{~mm}$ for $U_{S}$ of $4850 \mathrm{~m} / \mathrm{s}$, which results from an impact velocity of $500 \mathrm{~m} / \mathrm{s}$. All shock calculations were 
performed using standard equations of state and conservation equations [12]. Two rings were implemented as suggested by Bourne and Gray III to obtain optimal results [9], the inner ring and the outer rings had a width of $4 \mathrm{~mm}$ each. Samples without rings were placed $8 \mathrm{~mm}$ from the circumference of the flyer to compare the effectiveness of using the target holder as a momentum trap ring. Comparison between the two sample-holder configurations were performed from spall strength measurements and post-impact microscopy of the spall planes.

\section{Results and discussion}

The mechanical properties of the tested materials were inferred using ultrasonic techniques and are tabulated in Table 2, showing the materials have similar acoustic properties.

Table 2. The mechanical properties of the used materials.

\begin{tabular}{|c|c|c|c|c|}
\hline Material & $\begin{array}{c}\text { Density } \\
\left(\mathrm{kg} / \mathrm{m}^{3}\right)\end{array}$ & $\begin{array}{c}\text { Longitudinal } \\
\text { sound speed } \\
\left(c_{l}\right) \\
(\mathrm{km} / \mathrm{s})\end{array}$ & $\begin{array}{c}\text { Shear } \\
\text { sound } \\
\text { speed }\left(c_{s}\right) \\
(\mathrm{km} / \mathrm{s})\end{array}$ & $\begin{array}{c}\text { Bulk } \\
\text { sound } \\
\text { speed }\left(c_{o}\right) \\
(\mathrm{km} / \mathrm{s})\end{array}$ \\
\hline LDX2404 & 7700 & 5.883 & 3.302 & 4.351 \\
\hline LDX2101 & 7730 & 5.812 & 3.337 & 4.480 \\
\hline
\end{tabular}

EBSD examination for the as-received LDX2404 reveals that the material has $50 / 50$ of austenite phase (Face Centred Cubic structure (FCC)) and ferrite phase (Body Centred Cubic structure (BCC)) as shown in Fig. (3 a). The misorientation diagrams of both phases and as a bulk material are shown in Fig. ( $3 \mathrm{~b}$ and c). Fig. (3 b) shows the misorientation diagrams including grains and other structures (sub-grains, dislocation walls and twins), the dominant misorientation angle for the austenite phase and the bulk material is $60^{\circ}$. This misorientation angle explains that the annealing twins $\left(\sum 3\right)$ exist in the FCC phase. The BCC phase has predominantly Low Angle Grain Boundaries (LAGB) with misorientation angles less than $15^{\circ}$. The misorientation between the parent grains was explored in Fig. (3 c). The FCC phase's grains have misorientation angles varying from $25^{\circ}$ to $60^{\circ}$ when the annealing twins are excluded, typical of a randomly textured FCC material following the standard McKenzie distribution [4] . The BCC phase's grains, on the other hand, show a wider distribution of misorientation angles between $5^{\circ}$ to $60^{\circ}$. The ferrite phase has an average size of about $26 \mu \mathrm{m}$, the grain size of austenite phase is about $20 \mu \mathrm{m}$ and $18 \mu \mathrm{m}$ when the annealing twins excluded and included in the calculations, respectively.

Table (3) lists the testing conditions of the three experiments done in this study.

Fig. (4) shows the free surface velocities of the samples tested at the lowest and highest impact velocities. The dynamic yield stresses (Huguenot Elastic Limit-HEL) were estimated using Eq. (2) to be $2.04 \mathrm{GPa}$ for test No.1 and test No. 3.
Table 3. Conditions of the performed experiments.

\begin{tabular}{|c|c|c|c|c|}
\hline $\begin{array}{c}\text { Test } \\
\text { No. }\end{array}$ & $\begin{array}{c}\text { Impact } \\
\text { velocity } \\
(\mathrm{m} / \mathrm{s})\end{array}$ & $\begin{array}{c}\text { Flyer } \\
\text { thickness } \\
(\mathrm{mm})\end{array}$ & $\begin{array}{c}\text { Targets } \\
\text { holder } \\
\text { thickness } \\
(\mathrm{mm})\end{array}$ & $\begin{array}{c}\text { Peak } \\
\text { stress } \\
(\mathrm{GPa})\end{array}$ \\
\hline 1 & 255 & 1.432 & 2.842 & 4.54 \\
\hline 2 & 315 & 1.415 & 2.905 & 5.65 \\
\hline 3 & 446 & 1.255 & 2.620 & 8.20 \\
\hline
\end{tabular}

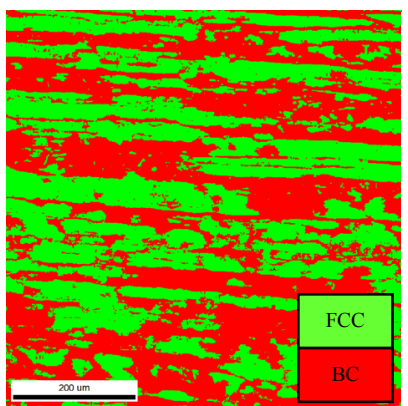

(a)

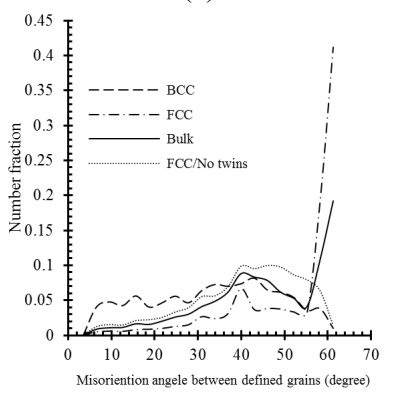

(c)

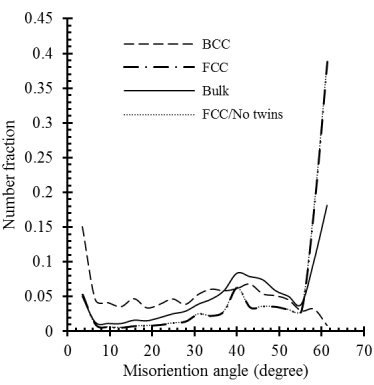

(b)

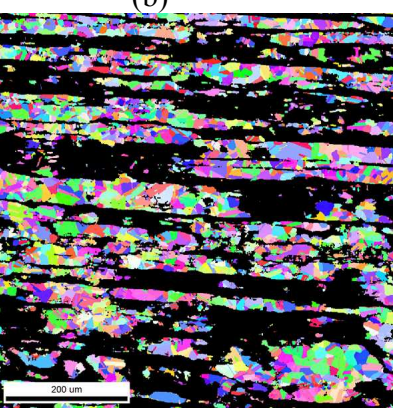

(d)



(e)
Fig. 3. EBSD results for the tested LDX2404 for the normal plane (a) Phases map, (b) misorientation diagrams for grains and substructures, (c) misorientation diagrams for defined grains only, (d) orientation map of the austenite phase, (e) orientation map of the ferrite phase.

$$
H E L=\frac{\rho_{o} c_{l} u_{H E L}}{2}
$$

where $\rho_{o}, c_{l}$ and $u_{H E L}$ are the initial density, the longitudinal sound speed and the free surface velocity at the precursor front. The existence of pull-back signals in the free surface velocity profiles for both conditions reveals the occurrence of a spall damage for both conditions. The spall strength was estimated using Eq. (3) at $2.68 \mathrm{GPa}$ for test No.1 and test No. 3. 


$$
\sigma_{\text {spall }}=\frac{\rho_{o} c_{o} \Delta u}{2}
$$

where $\rho_{o}$ and $c_{l}$ are the initial density, the bulk sound speed, respectively. $\Delta u=u_{\max }-u_{\min }$ where $u_{\max }$ and $u_{\min }$ are the maximum and the first minimum free surface velocities, respectively. It is clear that there is no effect for momentum trap rings on the velocity profiles (i.e. the usage of rings does not affect the spall strength). However, the recovered samples show significant differences in the damage content (Fig. (5)). This indicates clearly that radial release waves influences take place after the occurrence of spall damage.



Fig. 4. Free surface velocities of the tested samples.

The spall strength of LDX2404 is higher than LDX2101, we previously determined the spall strength of LDX2101 to be about $2.30 \mathrm{GPa}$ for the normal direction at a peak stress of $4.20 \mathrm{GPa}$ [13]. Moreover, the HEL of LDX2404 is higher than the HEL of LDX2101 reported by Silverstein and Eliezer ${ }^{[14]}$. This difference could be attributed to the grain size of LDX2404 being significantly smaller than the grain size of LDX2101 for both phases. Additionally, the content of nickel in LDX2404 is about two times higher, where increasing nickel content can enhance the mechanical properties based on the solid solutions strengthening mechanism [15-16]. There is no difference in the spall strengths for the reported conditions with an increase in the dynamic pressure by about $81 \%$ between test No.1 and test No.3, this response is completely different than the response of LDX2101 for a similar peak stresses, where an increase in the spall strength by about $27 \%$ was reported by Silverstein and Eliezer [14]. Therefore, further investigation is required to understand this.

Fig. (5) shows the optical images (processed images using Image $J \circledR$ software) for the diametral plane of the recovered samples. By comparing the amount of damage (black regions) in Fig. (5), the amount of damage is higher for samples surrounded with rings. The difference might not be clear for Fig. ( 5 a and 5 b), this because the small amount of damage at this low speed. For the test No. 3 (i.e. Fig ( 5 e and 5 f)), the sample without rings experienced clearly less damage compared with the sample with rings. This difference is attributed to the effects of radial release waves, which usually compress the sample during a shock experiment [17]. This compressive load could lead to closing some of the spall damage locations. As a result, using momentum trap rings is not essential to determine the spall strength; however, it is crucial when the microstructural examination for the recovered samples is required.
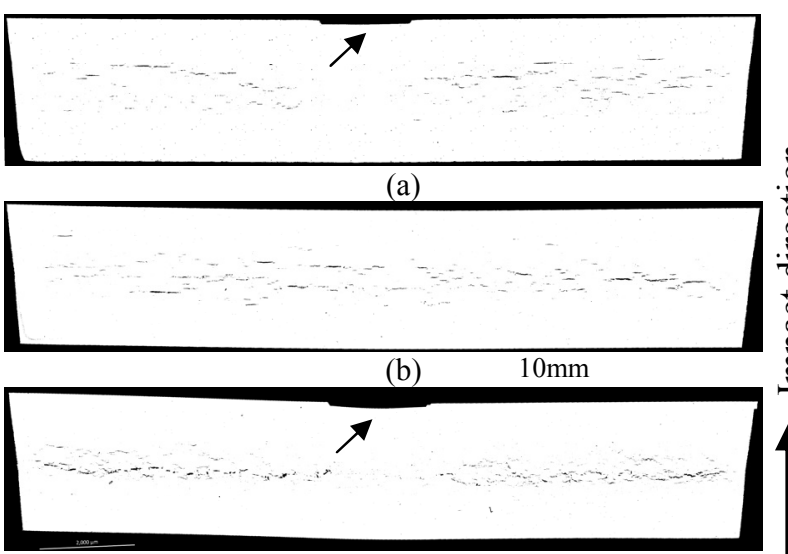

(c)

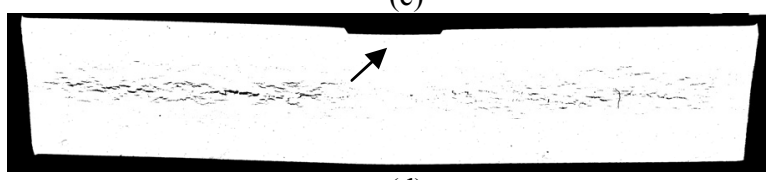

(d)

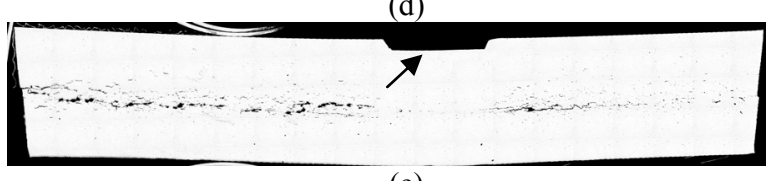

(e)

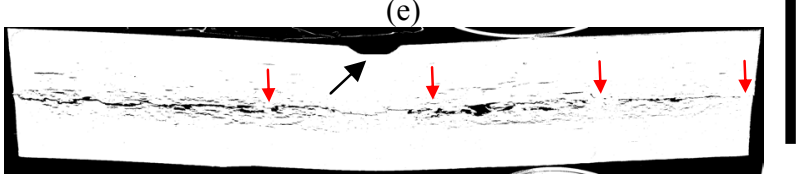

(f)

Fig. 5. Processed OM images of the recovered samples (a) test No.1 without rings, (b) test No.1 with rings, (c) test No.2 without rings, (d) test No.2 with rings, (e) test No.3 without rings and (f) test No.3 with rings.

The spall damage in the tested conditions varies clearly with impact velocity. For test No. 1 (Fig. 5 a and 5 b) and 2 (Fig. $5 \mathrm{c}$ and $5 \mathrm{~d}$ ), the spall damage remained in the incipient, or nucleation, stage; but, the amount of the damage for test No. 2 has clearly undergone growth and some coalescence. For test No.3, because the effect of radial release waves is significant, the sample without rings contains a mix of spall nucleation, growth, and coalescence. However, the sample with rings for the same shock conditions has advanced stages of void growth and coalescence, bordering a full spall fracture plane which is continuous along the diameter of the sample except for a few locations marked with red arrows in Fig. (5 f).

It is worth mentioning that the indention marks at the back surface of the recovered samples pointed by black arrows results from impacting the samples with the PDV probes. This impact causes a compression load in the middle of the sample which leads to reduce the width of the spall damages.

Fig. (6 a) shows the EBSD map for the recovered sample of test No.1 with rings (i.e. Fig. 5 b) around a region containing spall damage. It is clear that the crack orientation is parallel to the phase boundaries and normal 
to the impact direction. Furthermore, the spall damage is mainly accommodated by the ferrite phase, and propagates transgranularly (inside the grains away from the boundaries). The austenite islands inside the ferrite matrix work as obstacles for the crack propagation where the fracture path moves around the grain as shown in Fig. (6 a), indicated by blue arrows. This observation is consistent to the spall damage profile of LDX2101 shocked along the normal direction we reported in a previous study ${ }^{[13]}$. The effect of grain orientations on the spall damage coalescence was studied by investigating the Taylor factors for the ferrite phase with the loading direction parallel to the impact direction as shown in Fig. $(6 \mathrm{~b})$. This figure shows austenite grains with white colour while crack path with black colour.

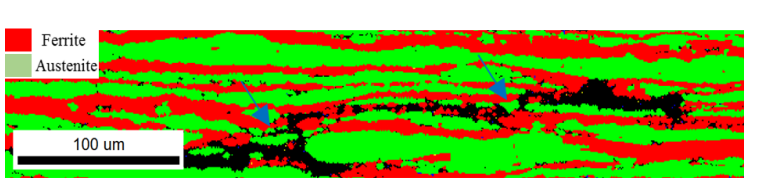

(a)



(b)

Fig 6. (a) phase map of the recovered sample of test No.1 (b) Taylor factor map of the ferrite phase, austenite grains have white colour and fracture path has black colour.

Fig. (6 b) shows that the crack path does not depend on the grain orientation (Taylor factor). A higher Taylor factor value means a higher external stress is required to activate slip systems. Crack location near the blue arrow in Fig. (6 b) explains that the crack took place with grains has a very low Taylor factor value (grains with blue and green colours have the lowest Taylor factor values). However, area close to the red arrow shows ferrite grain with the highest Taylor factor value experienced spall damage.

\section{Conclusions}

This paper investigated the spall strength of the newest commercial Lean Duplex Stainless Steel alloy 2404 (LDX2404) and the effect of radial release waves on the spall damage. Based on the presented results, the following points were concluded:

- Using double momentum trap rings is an effective approach to protect recovered samples from radial release waves.

- The spall strength magnitude is not affected by the radial release waves because radial release waves reach the sample after the spall occurrence.

- The spall strength and HEL of LDX2404 were estimated to be $2.68 \mathrm{GPa}$ and $2.04 \mathrm{GPa}$ for both of the testing conditions, respectively.
- The spall damage of the tested LDX2404 was accommodated mainly by the ferrite phase; it is parallel to the phase boundaries and normal to the impact direction.

- The void nucleation and void growth were not dependent on the grain orientation based on Taylor factor values.

Authors would like to acknowledge the support by the Air Force Office of Scientific Research under grant number FA2386-17-1-4095. The financial support of the Australian Commonwealth Government for the PhD study of the first author is also acknowledged.

\section{References}

1. A.A.H. Ameri, J.P. Escobedo-Diaz, M. Ashraf, and M.Z. Quadir: in Characterization of Minerals, Metals, and Materials, S. Ikhmayies, B. Li, J.S. Carpenter, J. Li, J.-Y. Hwang, S.N. Monteiro, M. Zhang, F. Donato, J.P. Escobedo-Diaz, Z. Peng, Y.E. Kalay, C. Ba, J. Kim, and G. Ramasis, eds., Sp, pp. 181-90 (2017)

2. M. Liljas, P. Johansson, L. Hui-Ping, Olsson, and C.-O. A.: Steel Res. Int., 2008, vol. 79, pp. 466-73.

3. Z. Zhang, H. Zhang, H. Zhao, and J. Li: Corros. Sci., vol. 103, pp. 189-95 (2016)

4. A.D. Brown, L. Wayne, Q. Pham, K. Krishnan, P. Peralta, S.N. Luo, B.M. Patterson, S. Greenfield, D. Byler, K.J. McClellan, A. Koskelo, R. Dickerson, and X. Xiao: Metall. Mater. Trans. A Phys. Metall. Mater. Sci., vol. 46, pp. 4539-47, (2015)

5. J.P. Escobedo, D. Dennis-Koller, E.K. Cerreta, B.M. Patterson, C.A. Bronkhorst, B.L. Hansen, D. Tonks, and R.A. Lebensohn: J. Appl. Phys., , DOI:10.1063/1.3607294.

6. A.L. Stevens and O.E. Jones: J. Appl. Mech., vol. 39, pp. 359-66 (1972)

7. J.P. Escobedo, E.K. Cerreta, and D. Dennis-Koller: , DOI:10.1007/s11837-013-0798-6

8 J.E. Field, S.M. Walley, W.G. Proud, H.T. Goldrein, and C.R. Siviour: Review of Experimental Techniques for High Rate Deformation and Shock Studies, vol. 30 (2004)

9. N.K. Bourne and G.T. Gray, III: Proc. R. Soc. A Math. Phys. Eng. Sci., vol. 461, pp. 3297-312 (2005)

10. G.T. Gray: High-Pressure Shock Compression Solids, pp. 187-215 (1993)

11. R.N. Orava and R.H. Wittman: in 5th Intenationa conference, High Energy Rate Fabrication, Denver, pp. 1-27 (1975)

12. P.J. Hazell: Armour: Materials, Theory, and Design, CRC Press (2016)

13. A.A.H. Ameri, J.P. Escobedo-Diaz, M.Z. Quadir, A. Brown, M. Ashraf, and P. Hazell: in AIP Conference Proceedings, 2017, p. Accepted.

14. R. Silverstein, D. Eliezer, B. Glam, D. Moreno, and S. Eliezer: J. Mater. Sci., vol. 49, pp. 4025-31 (2014)

15. G.E. Totten, L. Xie, and K. Funatani: Handbook of 
Mechanical Alloy Design, Marcel Dekker (2004)

16. A.A.H. Ameri, N.N. Elewa, M. Ashraf, and J.P. Escobedo-Diaz: Mater. Charact., vol. 131, pp. 32430 (2017)

17. G.T. Gray, P.S. Follansbee, and C.E. Frantz: Mater. Sci. Eng. A, vol. 111, pp. 9-16 (1989) 